\title{
LE FRISSON DE SAPPHO
}

\author{
DAVID BOUVIER \\ Faculté des Lettres \\ Université de Lausanne (Suíça)
}

\begin{abstract}
RÉSUMÉ: S'il est vrai que la compréhension d'une ouvre antique est toujours influencée et conditionnée, entre autres facteurs, par l'histoire des relais, traductions, interprétations, reprises et réappropriations qui ont assuré sa transmission et sa réception, ce constat prend une force exemplaire dans le cas de la poésie de Sappho. Parole féminine dans un monde culturellement dominé par le masculin, marquée du sceau de la différence, la poésie de Sappho a été expliquée autant qu'elle a été réinventée, récupérée et subvertie. Mais si sa poésie est indissociable de l'histoire complexe de sa réception, Sappho aussi s'approprie une tradition, voire des traditions, qu'elle contribue à transformer.
\end{abstract}

MOTS-CLEFS: Sappho; frisson; tressaillement; réception; tradition.

\section{Le frisson ( $\left.\phi \rho^{\prime} \kappa \eta\right)$ d'Hélène et le tressaillement ( $\left.\tau \rho \zeta \mu \circ \varsigma\right)$ de Sappho}

S'il est vrai que la compréhension d'une œuvre antique est toujours influencée et conditionnée, entre autres facteurs, par l'histoire des relais, traductions, interprétations, reprises et réappropriations qui ont assuré sa transmission et sa réception, ce constat prend une force exemplaire dans le cas de la poésie de Sappho. Parole féminine dans un monde culturellement dominé par le masculin, marquée du sceau de la différence ${ }^{1}$, la poésie de Sappho a été expliquée autant qu'elle a été réinventée, récupérée et subvertie. Comme si toujours il fallait atténuer la différence, dissiper la spécificité même de cette parole "trop" féminine, issue d'une poétesse dont on dénonce le caractère "instable" - pour reprendre

1 Sur les conséquences de ce constat, voir récemment les remarques de Yatromanolakis, 2007, 366-367. 
l'expression d'un témoignage péripatéticien masculin ${ }^{2}$. Les fragments qui nous restent de sa poésie ont chacun une histoire philologique différente, liée à des cheminements propres: mais toujours il faut se méfier d'une plume masculine qui cite une parole féminine. Ainsi quand le Pseudo-Longin cite l'ode 31, il faut prendre garde à la façon dont il encadre la citation et le commentaire des vers de Sappho par des citations des poèmes homériques, avec - on va le voir - un subtil parallèle entre le "frémissement ( $\left.\tau \rho \mu_{\mu \circ \varsigma}\right)$ " de peur des guerriers et celui de la narratrice de l'ode $31^{3}$.

Mais si la poésie de Sappho est indissociable de l'histoire complexe de sa réception, il faut aussi rappeler la dette de Sappho. Elle aussi s'approprie une tradition, voire des traditions, qu'elle contribue à transformer. Prenons l'exemple de l'ode 31 et limitons-nous plus précisément à ce "frémissement", pour traduire ainsi le terme $\tau p o ́ \mu o s$, qu'éprouve la narratrice aux vers 13-14. Symptôme privilégié

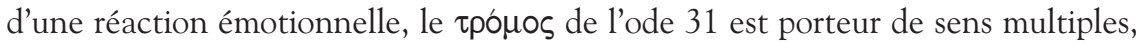
"tressaillement, tremblement, frisson, chaire de poule...". Comment traduire? Comment interpréter cette sensation? Sans oublier que les mots ont une mémoire dont Sappho hérite. C'est alors la poésie homérique qui offre le meilleur point de comparaison; mais l'interprète doit se prémunir contre la tentation d'établir des filiations directes entre des traditions poétiques parallèles et croisées ${ }^{4}$. Chez Homère, le $\tau p o ́ \mu o s$ est signe de peur ou de crainte. Quand Thétis apporte à Achille ses armes divines, c'est un "frémissement" ( $\tau \rho o ́ \mu \circ)$ qui saisit les Myrmidons à la vue du bronze. Voyant Achille, tel Arès, se lancer contre lui, Hector est saisi d'un

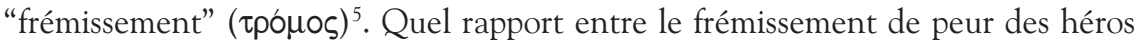
sur le champ de bataille et la narratrice de l'ode 31 saisie d'un frémissement à la vue d'une autre femme au rire désirable? Une femme peut-elle dans la poésie homérique susciter la peur ou provoquer chez ceux qui la regardent un "frémissement"? Oui, mais le mot qui désigne le frisson est alors différent; rappelons la dernière image de l'Iliade, quand, pleurant Hector, la plus belle des femmes grecques, Hélène, décrit le frisson qu'elle provoque chez tous ceux qui la regardent:

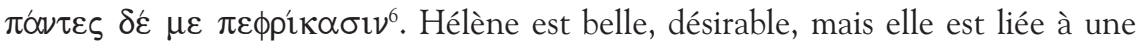

2 POxy. 1800, fr. 1 = Chamael. F 27 Wehrli et Ferrari, 2007, 166. Sur la réputation de Sappho dans l'Antiquité, Most, 1996, 11-9; Lefkowitz, 1996; Iriarte, 2007 et les pages introductives de Ragusa, 2005, 23-53.

3 Cf. infra.

4 Voir l'avertissement pertinent de Calame, 2005, 31-3.

5 Il. XIX.14-17 et Il. XXII.136. Cf. aussi infra. 
guerre et une histoire qui la rendent effrayante. Du moins est-ce l'opinion d'Achille. Pour qualifier Hélène, cause du conflit et du malheur de tous, il emploie un adjectif

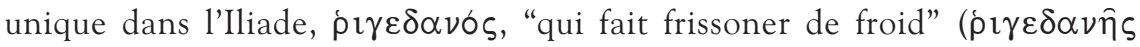

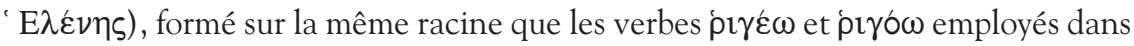
les poèmes homériques pour signifier le frissonnement de crainte ou de froid? LHélène devant qui "tous frissonnent" est-elle une beauté qui glace comme la crainte $^{8}$ ?

Frissonner devant une femme qui suscite désir et peur? Les références homériques invitent à penser que l'effet de la beauté d'Hélène était un thème volontiers exploité par les aèdes des traditions orales épiques. Il importe alors de s'interroger sur la culture de Sappho, sur le rôle dans sa formation d'une initiation à des traditions épiques dont elle se révèle imprégnée ${ }^{9}$. Mais sans aller si loin, je veux simplement dans cette étude m'interroger sur les interprétations de ce

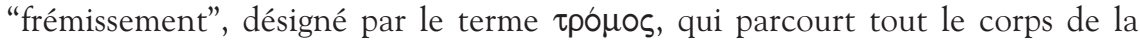
narratrice de l'ode 31, une narratrice que beaucoup ont identifiée à Sappho ellemême: symptôme d'un malaise amoureux, signe d'une expérience extatique, effet de la peur, de l'inspiration, marque d'une révélation? Chemin faisant, on verra

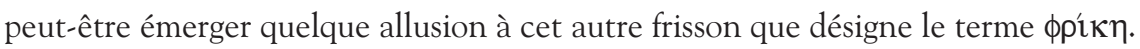

\section{Le pouvoir suggestif de l'ode 31}

Lode 31 a un pouvoir suggestif d'autant plus fort que Sappho a voulu sa polysémie. Mais deux millénaires et demi plus tard, c'est aussi l'histoire de sa réception qui transforme et complique le texte reconstruit par les éditeurs. Lode 31 nous est connue grâce à sa citation dans le traité du Sublime, (ci-après Пepì

6 Il. XXIV.775.

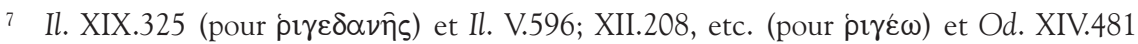
(pour ṕrócw).

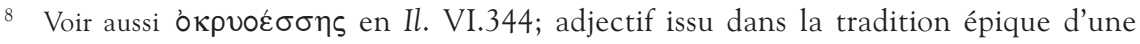
déformation de крvóєıৎ: "qui fait frissonner, d'un froid glacial”, employé pour qualifier la crainte en Il. IX.2; cf. Hainsworth, 1993, 68.

9 La question des rapports entre la poésie de Sappho et celle d'Homère fait l'objet d'un débat, rappelé par Calame, 2005, 31-3. Ferrari, 1986, 441-7 et Rissman, 1983, 66-118 offrent un matériel important pour repérer des échos ou parallèles; voir aussi Marcovich, 1972, 26. La thèse de Rissman est critiquée déjà par Fowler, 1986. Pour d'autres nuances, cf. Schrenk, 1994; Rosenmeyer, 1997; Steinrück, 1999; Bouvier, 2009. Larson 2010, 175-6 donne une bibliographie récente et montre que le débat n’est pas clos. 
v̛(ov૬). Mais ce texte, qui transmet les 4 premières strophes d'un poème qui devait être plus long, pose plusieurs problèmes que les témoignages parallèles ne permettent pas de trancher. Pour chaque vers les possibilités de compréhension -et donc les difficultés de traduction se multiplient. La narratrice évoque, à son "paraître"

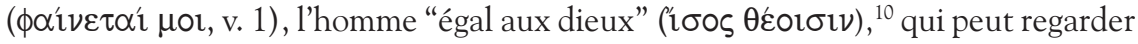
de près cette femme (interpellée à la deuxième personne, mais anonyme) à la voix douce et au rire agréable. "Cela" ( $\tau$ ) , dit la narratrice (v. 5-6), provoque la

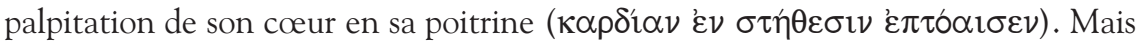
à quoi renvoie exactement le $\tau$ ó? Les interprètes divergent et s'opposent sur le référent exact de ce pronom anaphorique ${ }^{11}$. Est-ce le seul rire de la jeune fille? Son rire et sa voix? Le fait que le rire s'adresse à un homme? Faut-il reconnaitre un sentiment de jalousie et considérer que le $\tau$ to renvoie alors à l'intimité partagée de

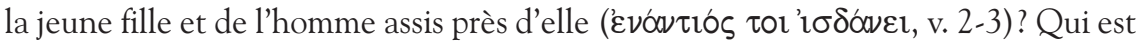
cette femme? Les traducteurs la disent “jeune”, mais le texte grec n'est pas si précis. Qui est-elle par rapport à la narratrice? Et cet homme est-il réellement présent? Un homme quelconque ou particulier? S'agit-il d'un mari, d'un fiancé, d'un visiteur? L'ode est-elle un épithalame? Le débat reste ouvert et sans doute est-il bon d'en préciser encore l'histoire.

Et la question se renforce au vers 7 où le texte est peu sûr: $\omega \varsigma$ rò̀ है $\zeta$

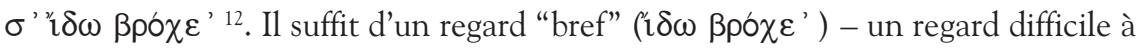
soutenir longtemps $-{ }^{13}$ vers la femme désignée par le pronom de la $2^{\mathrm{e}}$ personne $\left({ }^{\prime} \varsigma \sigma^{\prime}\right)^{14}$ pour que la narratrice éprouve une suite de sensations qu'elle décrit en un rythme qui semble s'accélérer et où Madame Dacier entendait cette "perte d'haleine qui arrive à tous ceux qui tombent peu à peu en pâmoison" ${ }^{\text {"15 }}$ la voix qui se brise, le feu léger qui court sous la peau, le regard qui s'aveugle, les oreilles qui

10 Je suis l'édition de Voigt, 1971. Pour un commentaire, cf. Hutchinson, 2001, 160-77 et toujours Degani \& Burzachini, 1977, 139-46, avec mise à jour dans Degani \& Burzachini, 2005; voir aussi Privitera, 1969b qui suit le texte de l'édition de Lobel - Page et donne un commentaire précieux.

11 Most, 1996, 28-9 et Prins, 1996, 39-42.

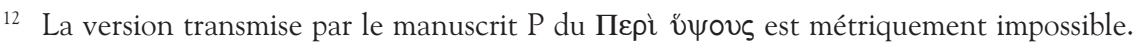

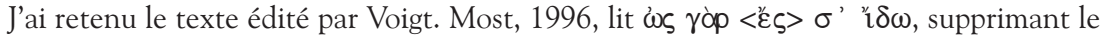
pronom.

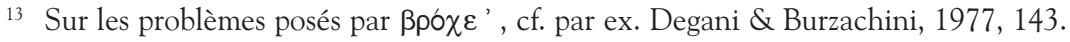

14 Si l'on accepte cette leçon pour le texte.

15 Les poésies d'Anacréon et de Sapho, traduites de grec en françois, avec des remarques, par Madame Dacier, Paris, chez D. Thierry \& C. Barbin, 424. 
bourdonnent, la sueur froide, le tremblement, le teint pâle et, enfin, l'impression de mourir... ${ }^{16}$ Autant de symptômes, réels ou métaphoriques, qui ont autorisé un nombre infini d'interprétations. Sentiment de jalousie ou amoureux? Ode nuptiale? Situation fictive ${ }^{17}$ Amour féminin ou masculin? Homosexuel ou hétérosexuel? Certains, comme Beattie, ont voulu supprimer le "tu" $\left(\sigma^{\prime}\right)$ du vers 7 pour affirmer que la narratrice tombait sous le charme de l'homme et non de la femme ${ }^{18}$. Amour chaste ou non? Madame Dacier, première traductrice française connue de Sappho, transforme ainsi le sentiment amoureux en sentiment d'amitié ${ }^{19}$, répliquant à son père, Tanneguy Le Fèvre ${ }^{20}$, austère érudit protestant qui fut l'un des tout premiers à admettre ouvertement en France l'homosexualité de Sappho.

\section{Valeurs et significations multiples d'un "frémissement"}

La polysémie de l'ode 31 touche autant l'ensemble que le détail des vers.

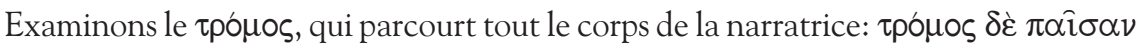
ø̌ $\gamma \rho \varepsilon ı$, une phrase où les allitérations laissent entendre un "roulement de liquides" qui résonne dans l'adjectif $\chi \lambda \omega \rho o \tau \varepsilon p \alpha$ qui suit et introduit le symptôme suivant ${ }^{21}$. Les traductions révèlent une série d'hésitations dues aussi au champ sémantique élargi couvert par ce terme: "tremblement", "frisson", "frémissement". Le LSJ

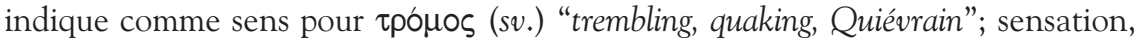
explique le LSJ, provoquée "from fear" (renvoi à Il. XIX 14), "from love (renvoi à Sapph. 31.13; et pour l'emploi au pluriel, "shiverings”, à Hp. Coac. 92); ou encore "from cold" 22 . Les traducteurs de l'ode jouent sur la variété de ces sens. Le

16 Pour les maints problèmes philologiques que posent ces vers, cf. les commentaires cités note 10 .

17 Cf. les arguments de Lasserre, 1989, 147- 152.

18 Privitera, 1969b, 40.

19 Paradiso, 2003, 61 et aussi DeJean, 1994, 56.

20 Voir DeJean, 1994, 85 et Most, 1996, 19.

21 Sur les allitérations dans l'ode 31, cf. Segal, 1996, 65-6. L'analyse de Neri \& Citti, 2003,

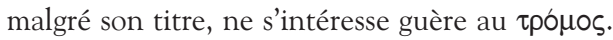

22 Le Bailly (s.v.) traduit par: "tremblement, frisson" et n'indique comme cause que la peur

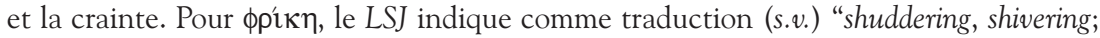
a mild form of ṕîos, cold fit before fever" et ajoute le sens de frisson d'horreur (E. Ph. 1284); le Bailly (s.v.) traduit par: "frissonnement" et indique comme emplois plus particuliers: "frisson de la peau", provoqué par la frayeur, le froid ou la fièvre, ou le respect d'une divinité (Hdt. VI 134; S. OT 1306). 
frémissement relève de la crainte dans les traductions de Ronsard ("je tremble tout de crainte") et de Rémi Belleau plus affirmatif encore: "je suis en chasse à l'horreur, à la peur..."23. Au sentiment de frayeur s'oppose, dans d'autres traductions, l'expression d'un sentiment extatique, en rapport avec une expérience de possession. Chez Boileau, qui traduit l'ode 31 dans sa traduction du traité Du Sublime et qui se permet de supprimer des expressions pour en ajouter d'autres, le frémissement est explicité par une sensation de transport langoureux: "Je n'entends plus, je tombe en de douces langueurs; / Et, pâle, sans haleine, interdite, éperdue, / Un frisson me saisit, je tremble, je meurs." ${ }^{24}$ Parfois encore, la traduction exploite un registre plus technique, voire plus médical: "Une fièvre secrète me consume lentement, / Je ne vois plus rien, ni n'entends. / Mes oreilles bourdonnent, / Mon corps est trempé de sueurs froides, / Je tremble de tout mon être." ${ }^{25}$ Enfin, et sans être exhaustif, il

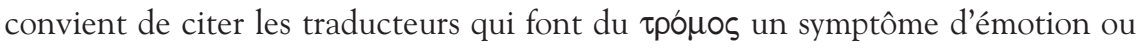
d'abandon amoureux: "Partout sur moi coule la sueur, un frisson / Toute me perd, plus verte que le gazon / Je suis, défaillante et sans force à peu près/ Je parais changée." ${ }^{26}$ Souvent, un titre annonce la ligne d'interprétation: "A une femme aimée", "A la bien aimée", "A l'aimée"27.

Dans l'Antiquité déjà, les courants divergent et les "symptômes" de l'ode 31 inspirent des réécritures ou des réappropriations différentes, qui s'opposent parfois consciemment. Dans son Idylle des Magiciennes, Théocrite associe à la passion amoureuse de son personnage une pathologie clairement issue de l'ode 31: "Connais mon amour, d'où il est venu, auguste Séléné. Tout entière je fus plus glacée que neige; sur mon front la sueur ruisselait pareille aux gouttes de rosée; je ne pouvais proférer aucun son, pas même des balbutiements comme ceux que, dans leur sommeil, les enfants adressent à leur mère; tout mon corps devint raide, à l'égal

23 Pierre de Ronsard, "Les Amours de Marie", Le Second Livre des Amours commenté par Remi Belleau du Perche, Paris, 1560 et Remi Belleau, Les Odes d'Anacréon Téien traduites de Grec en François, ensemble quelques petites odes de son invention, Paris 1556. Les deux traductions sont commodément regroupées, avec d'autres, dans Brunet, 1998, 25-7.

24 Boileau, Euvres complètes, édition établie par Françoise Escal, Paris, Gallimard (Bibliothèque de la Pléiade), 1966, 357 et pour le commentaire de Boileau, 416; cf. aussi DeJean, 1994, 77.

25 Danièle Calvo Platero, citée in Brunet, 1998, 121.

26 Jym, Sappho de Mitylène réhabilitée, Fragments authentiques de ses poèmes d'après Bergk, Boissonade, Lemerre et Diehl, traduction équirythmique, Angers 1937.

27 Voir les traductions d'Emile Deschanel, M. Redarez-Saint-Remy, Joseph Boulmier, etc. 
d'une poupée de cire. Connais mon amour..."28. C'est l'art et la force de Théocrite de réinventer un récit qui garde, pourtant, la marque de sa référence. On notera que le poète hellénistique a substitué au frémissement une sensation de froid glacial. Mais il s'agit d'un effet de l'amour. A l'opposé, dans la tradition de la poésie hexamétrique latine, Lucrèce se souvient des vers de Sappho pour les associer à une sensation de peur. Dans le De reum nature qui commence par une invocation à Vénus, Lucrèce aborde au livre III la question de l'âme et de la peur de la mort; question qui fut l'objet de la philosophie de Platon, mais surtout d'Epicure dont Lucrèce se fait le successeur ("te imitai aveo", III.6). Il faut chasser cette peur de la mort qui "pénètre jusqu'au fond de l'homme" et qui affecte l'âme ("anima") et l'esprit ("animus") simultanément: "Mais lorsqu'une crainte plus violente vient bouleverser l'esprit, nous voyons l'âme entière s'émouvoir de concert dans nos membres; et sous l'effet de cette sensation les suées et la pâleur se répandre sur tout le corps, la langue bégayer, la voix s'éteindre, la vue s'obscurcir, les oreilles tinter, les membres défaillir ("succidere artus"); enfin à cette terreur de l'esprit nous voyons l'homme succomber" ${ }^{\prime 2}$. Dans la liste de ces symptômes qui renvoient à l'ode 31, le frémissement manque remplacé par la défaillance des membres ("succidere artus") ${ }^{30}$. Cette disparition du frémissement, comme le remarque justement $\mathrm{O}$. Thévenaz dans une thèse récente, reste à comprendre tant il est vrai que, parmi les symptômes, le frémissement est le plus propice à expliquer la crainte. Que le frémissement soit supprimé dans une version qui veut insister sur une interprétation érotique des symptômes est compréhensible ${ }^{31}$, mais il l'est moins chez Lucrèce. Entre frisson de peur et frémissement amoureux, les Anciens déjà ouvraient, à travers leurs reprises ou échos à l'ode 31, deux interprétations du $\tau \rho \dot{\mu} \mu \varsigma^{32}$. Allons plus loin.

28 Theoc. II 105-111 (trad. Ph.-E. Legrand). Sur la réception de Sappho à l'époque hellénistique, cf. le travail important de Acosa-Hugues, 2010, 62-105 (dont j'ai pris connaissance trop tardivement pour m'y référer plus longuement).

29 Lucr. III.152-8 (trad. A. Ernout). Voir aussi Sen. Troad. 486-8.

30 Cf. Thévenaz, 2010, 69, (thèse inédite soutenue à l'Université de Lausanne, en juin 2010, et dont on attend la publication avec impatience).

31 Comme dans le poème 51 de Catulle.

32 Double lecture déjà relevée par Privitera, 1969b, 68. 


\section{Le médecin et la pulsation du frémissement amoureux}

L'une des réappropriations les plus étonnantes de l'ode 31 dans l'Antiquité se trouve dans une série d'anecdotes qui relatent un diagnostic médical relatif à un mal singulier. Au Ir siècle de notre ère, Plutarque raconte dans la Vie de Démétrios comment Erasistrate, médecin prestigieux de Séleucos $1^{\text {er }}$, s'inspira de Sappho pour l'un de ses diagnostics les plus délicats. Accablé de chagrin, Antiochos, fils de Séleucos, se laissait dépérir attendant la délivrance de la mort. Erasistrate, chargé de soigner le jeune homme, devine une passion amoureuse. Il s'installe dans la chambre du malade et le surveille, observant surtout "les parties et les mouvements

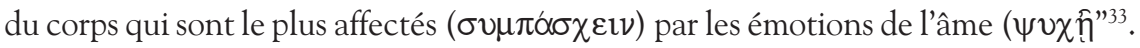
Or, chaque fois que Stratonice, la belle mère d'Antiochos, deuxième épouse de Séleucos, entre, seule ou accompagnée, le médecin vérifie - poursuit Plutarque "tous les symptômes décrits par Sappho: perte de la voix, rougeurs enflammées, obscurcissement de la vue, sueurs soudaines, désordre du pouls, et à la fin, quand

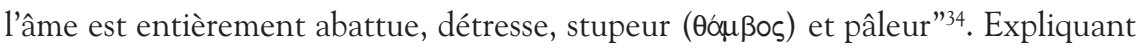
à Séleucos la violence et la nature du mal de son fils, Erasistrate, aussi fin psychologue que médecin, le conduit à admettre qu'Antiochos doit épouser la femme qu'il désire si ardemment, fût-elle la propre femme du roi ${ }^{35}$.

Dans cette version, Plutarque reconnaît l'autorité de Sappho comme poétesse par excellence de la passion amoureuse, une autorité que Platon reconnaissait déjà ${ }^{36}$. Sappho inspire Erasistrate dans son diagnostic d'une passion amoureuse qui a pris l'apparence d'une maladie; l'“amour", "̌p $\omega \varsigma$, est en effet dans cet épisode une "affection", $\pi \alpha \dot{\alpha} \theta \circ \varsigma$, qu' Antiochos combat ( $\tau \hat{\omega} \pi \dot{\alpha} \theta \varepsilon \varepsilon$

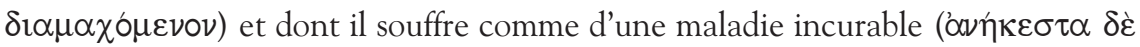

33 Plu. Demetr. 38.3.

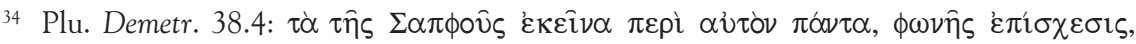

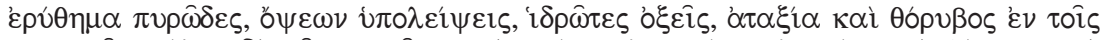

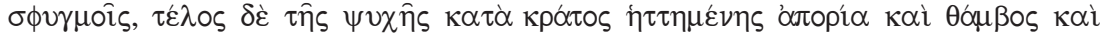

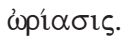

35 Les historiens se sont interrogés sur la vraisemblance de ce récit. Sans remettre en question le mariage de la reine avec son beau-fils (vers 293 av. J.-C.) - mariage qui peut trouver une explication politique - ils sont plus hésitants à admettre l'anecdote de la passion amoureuse. Quoi qu'il en soit, le motif connut un succès certain dans les écoles de rhétorique: il permettait d'argumenter sur la passion comme maladie de l'âme, sur la relation père - fils, sur la figure de la belle-mère. Voir à ce propos Lightfoot, 2003, 374.

36 Pl. Phædr. 235c3. 
vo $\sigma \varepsilon i v)^{37}$. Plutarque ne mentionne pas le frémissement, $\tau \rho o ́ \mu \circ \varsigma$, parmi les symptômes observés par Erasistrate. Mais Madame Dacier, dans son édition des Poésies d'Anacréon et de Sapho, observait avec finesse que Plutarque appelle $\theta \alpha ́ \mu \beta$ os ce que Sappho désigne par le terme $\tau \rho o ́ \mu \varsigma^{38}$. Plus qu'une simple substitution, il y a ici déplacement.

Faut-il s'étonner de ce déplacement qui transforme le "frémissement" en "stupeur admirative"? Ce n'est qu'un indice des multiples resémantisations dont le

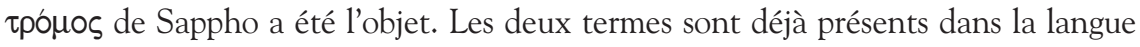
homérique où ils possèdent des valeurs sémantiques clairement distinctes. Le $\tau \rho o ́ n o \varsigma$ - on le verra - signifie un tremblement, un frémissement, toujours lié à la

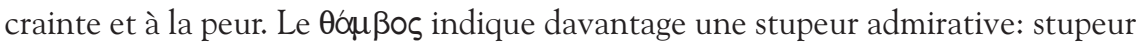
admirative des Troyens et des Achéens qui voient Athéna venir sur le champ de bataille; même stupeur des deux armées devant Ménélas et Paris prêts pour le duel qu'ils vont se livrer; cette stupeur admirative des spectateurs se transformerait à

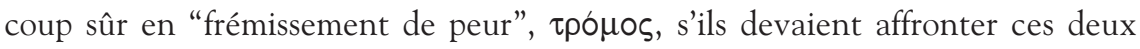
combattants $^{39}$. Surtout, le $\theta \alpha ́ \alpha \beta$ os marque l'étonnement admiratif d'Achille découvrant Priam, à ses genoux, qui le supplie de lui rendre son fils (Il. XXIV.482-4); étonnement qui deviendra bientôt "admiration" lorsque les deux hommes assis face

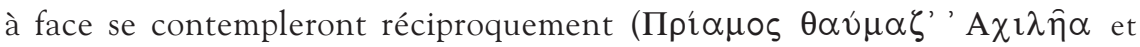

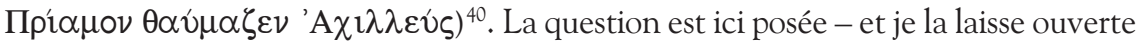

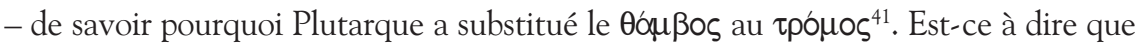

37 Cf. 38.2.4-5; cf. aussi 38.2.8 et 6.1. Pour d'autres variantes de ce récit et sur l'autorité médicale du diagnostic, cf. Mesk, 1913; Robiano, 2003 et les remarques de Lightfoot, 2003, 375-8 et 381 .

38 Les poésies d'Anacréon et de Sapho, traduites de grec en françois, avec des remarques, par Madame Dacier, Paris, chez D. Thierry \& C. Barbin, 425.

39 Il. IV.79 et Il. III.342.

40 Il. XXIV.629 et 631. Voir aussi Il. XXIII.815 et Od. III.272. Voir aussi, dans la perspective qui m'intéresse, Pl. Phaedr. 254c.

41 Remarquons que d'autres versions de l'anecdote oublient plus nettement encore le "tremblement". Dans la version, en latin, de Valère Maxime, il y a hésitation sur le statut du savant qui identifie le mal d'Antiochos, soit le médecin Erasistrate, soit l'astronome Leptine. Valère Maxime est plus précis qu'Appien, mais les symptômes qu'il mentionne ne renvoient pas non plus à Sappho. En présence de Stratonice, Antiochos rougit et sa respiration devient plus pressée; ce sont alors les battements du pouls qui permettent d'identifier le mal; Val. Max. 5-7ext.: "intrante enim Stratonice et rursus abeunte brachium adulescentis dissimulanter adprehendendo modo uegetiore modo languidiore pulsu uenarum conperit cuius morbi aeger esset”. 


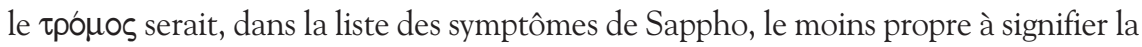
passion amoureuse? Ou plutôt, au contraire, que Plutarque a considéré la stupeur

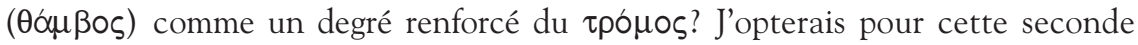
hypothèse.

Dans son De Dea Syria, rédigé dans la seconde moitié du IIe siècle de notre ère, Lucien, qui évoque le même épisode, n'hésite pas à réintroduire le terme

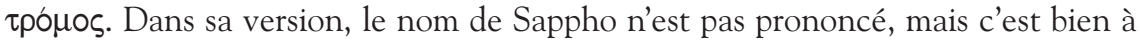
l'ode 31 que sont empruntés les symptômes $(\sigma \eta \mu \eta \dot{\imath} \alpha)$ du mal amoureux qui ronge Antiochos. Erasistrate met sa main droite sur le cœur du jeune homme tandis qu'il fait défiler ses proches devant lui: "à l'arrivée de sa belle-mère, sa peau change

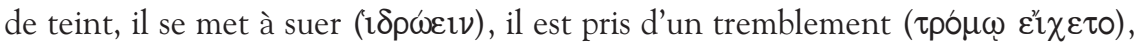
son cœur palpite. Ces réactions révèlent son amour au médecin" ${ }^{42}$. Faut-il franchir un pas de plus et penser que la médecine s'est approprié l'observation de Sappho pour faire le diagnostique d'une maladie à part? Lucien est, sur ce point plus clair que Plutarque; la maladie d'Antiochos "n'est pas une maladie" ${ }^{43}$, même s'il a fallu un médecin.

Dans la version d'Appien qui ne fait aucunement allusion aux vers de Sappho, l'amour est bien une "affection", $\pi \dot{a} \theta$ os, qui rend le jeune homme malade

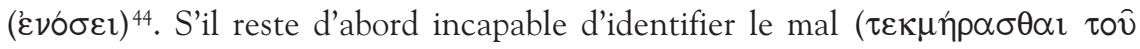
$\pi$ ó(Oous), Erasistrate conjecture finalement qu'il s'agit "d'une maladie de l'âme"

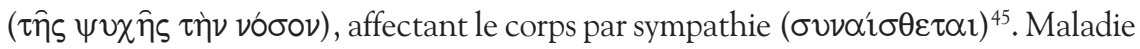
ou non? Galien viendra trancher le débat de façon catégorique en rappelant que la passion amoureuse n'est pas une maladie qui relève de l'art médical, mais que le bon sens suffit pour la diagnostiquer: "Ce n'est pas en regardant voler des corbeaux ou des corneilles qu'Erasistrate surprit l'amour du jeune homme, pas plus qu'il ne perçut des pulsations artérielles révélant de l'amour chez le jeune homme, contrairement à ce qu'ont écrit certains, car il n'y a pas de pouls particulier

42 Luc. Syr. D. 17.24; pour une autre occurrence de cette formule, cf. Zonar. s.v. ¿ $\xi \varepsilon 1 \sigma \tau \eta \dot{\kappa \varepsilon \varepsilon . ~}$

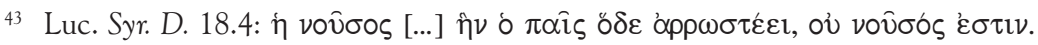

44 App. Syr. 309.4-5.

45 App. Syr. 310.1-3. Examinant Antiochos, Erasistrate ne constate, dans la version d'Appien, qu'une agitation plus grande du malade en présence de Stratonice; les symptômes particuliers ne sont pas énumérés; et le médecin conclut que c'est bien l'amour

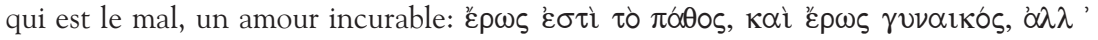

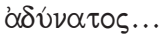




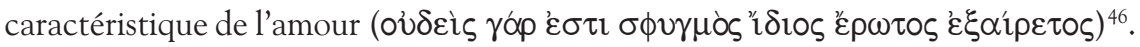
Posant la question des compétences qui permettent de diagnostiquer les affections de l'âme, Galien (auteur par ailleurs d'un traité Du tremblement, de la palpitation, de la convulsion, du frisson ${ }^{47}$ ) ignore complètement Sappho (contrairement à Plutarque) et nous invite à réfléchir sur toute une tradition qui a voulu voir dans les vers 5-16 de l'ode 31 les effets d'un malaise pour assimiler la passion amoureuse à une

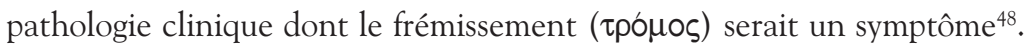

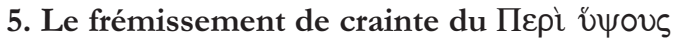

Signe pathologique ou non? Frémissement de peur ou frissonnement d'extase? L'histoire de la réception de l'ode 31 est, d'un point de vue philologique,

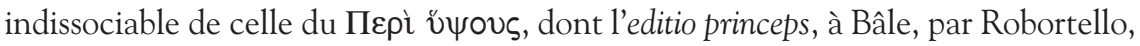
remonte à $1554^{49}$. Limportance de ce traité fut d'emblée évidente et Paul Manuce le rééditait l'an suivant à Venise, avant de s'en inspirer pour écrire son Discorso intorno all'ufficio dell'oratore. On sait que Fulvio Orsini, probablement le premier traducteur latin, fit connaître le texte à Michel-Ange ${ }^{50}$. Jusqu'aux découvertes

46 Gal. in Hipp. Progn. I. 18b40.4-7 Heeg (=CMG V 9.2, pp. 206.218). Cf. aussi Gal. De Praecog. 6 (CMG V 8.1, pp. 100-5).

47 G. Kühn, Claudii Galeni opera omnia, vol. 7. Leipzig: Knobloch, 1824 (repr. Hildesheim: Olms, 1965), 584-642.

48 Si l'on examine, par ailleurs, les textes médicaux anciens de la collection hippocratique, les symptômes évoqués par Sappho reviennent ici et là et certains rapprochements plus précis sont également possibles. Dans le traité des Affections internes, plusieurs des symptômes décrits dans l'ode 31 sont mentionnés dans le même ordre à propos d'une maladie fréquente mais non nécessairement mortelle (Sur les affections internes, 49). Di Benedetto n'hésite pas à penser que ce traité, postérieur à Sappho d'au moins deux siècles, remonte à une tradition antérieure dont la poétesse grecque se serait inspirée (Di Benedetto, 1985). Faut-il penser que Sappho a emprunté, la première, ses symptômes à la médecine avant que celle-ci ne les lui reprenne? Mais pourquoi exclure l'influence inverse quand le témoignage de Plutarque montre un médecin instruit par une ode poétique? Le plus intéressant est alors d'observer que les mêmes symptômes décrivent, d'un domaine à l'autre, des affections différentes. Voir aussi maintenant, Ferrari, 2007.

49 Sur l'attribution et datation de ce traité, cf. Mazzucchi, 1992, XXVII-XXXIV.

50 Mattioli, 1988, 109-112 (avec des indications bibliographiques) et 15-27. La première traduction en italien (vulgaro), restée inédite, fut sans doute celle de Niccolò da Falgano, tandis que la première publiée est celle de Niccolò Pinelli, en 1639. En France, après avoir été intégrée, dans la deuxième édition des Anacreontis et aliorum lyricorum aliquot 
papyrologiques d'Oxyrhynque, publiées à partir de 1898, ce traité de rhétorique, conservé par le Parisinus 2036 et ses apographes, fut le seul document, en notre possession, à citer l'ode 31. Curieusement l'influence de ce traité sur la compréhension de l'ode 31 n'a pas reçu toute l'attention nécessaire. Il faut cependant reconnaître que, dans la tradition française au moins, durant la Renaissance et jusqu'à Boileau, la réception de l'ode 31 a surtout subi l'influence du carmen ${ }^{51}$ de Catulle et de l'épitre fictive composée par Ovide dans sa quinzième Héroïde5 1. La situation change dans le dernier quart du XVII siècle quand Boileau traduit le Пвpi v̌yous et s'enthousiasme pour son auteur (que Boileau nomme Longin), lui accordant d'être "lui-même le grand sublime qu'il dépeint" ${ }^{\text {" Le Peudo- }}$ Longin, dont le jugement fait autorité, devient alors le meilleur avocat de la poésie de Sappho, tout en influençant, par ailleurs, radicalement l'interprétation de l'ode.

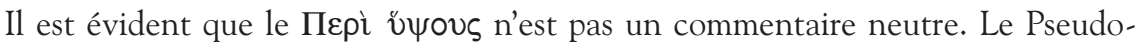
Longin choisit et découpe les citations qui servent son propos; plus encore, il sait, par un subtil travail de juxtaposition, les éclairer les unes par les autres pour faire ressortir des contrastes importants.

Au début du chapitre $X$, le Pseudo-Longin annonce vouloir examiner une

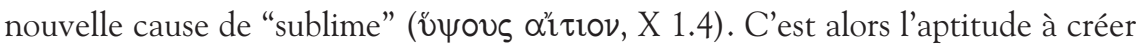
une unité cohérente à partir d'éléments divers qu'il va décrire en se référant à Sappho: "Puisque, à toute chose sont attachées, par nature, diverses parties inhérentes à sa substance, le principe du sublime ne consisterait-il pas nécessairement, pour nous, dans le fait de toujours choisir, parmi les éléments inhérents, ceux qui sont le plus appropriés et de savoir, en les assemblant les uns les autres, en faire comme un seul corps. En effet, tant le choix des arguments que l'accumulation des expressions choisies exercent un attrait sur l'auditeur" ${ }^{\text {"53. Et le }}$

poetarum odae d'Henri Estienne en 1556, l'ode 31 inspire d'abord un sonnet de Louise Labé est imitée avant d'être traduite une première fois en français par Rémi Belleau en 1556.

51 DeJean, 1994, 40-44.

52 Hertz, 1973, 292. Sur Пвpi úuovs et Sappho, cf. aussi Prins, 1996, 48-51. Voir aussi, passim, Michel, 1976.

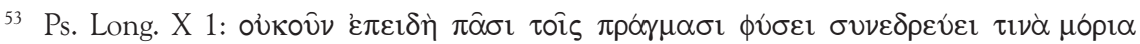

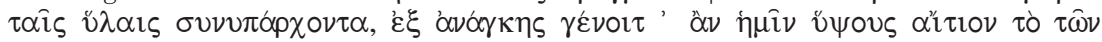

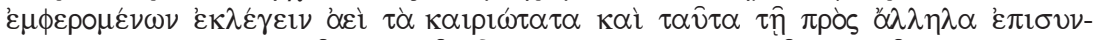

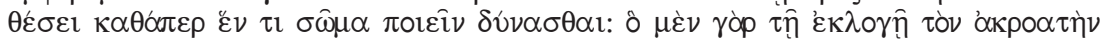

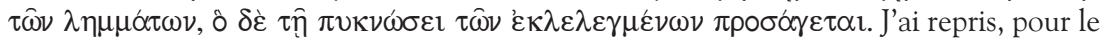

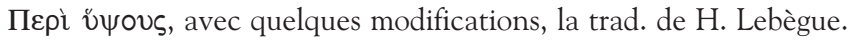


Pseudo-Longin poursuit pour indiquer que c'est là précisément ce que fait Sappho dans l'ode 31, lorsqu'elle décrit "les souffrances adhérentes aux fureurs amoureuses

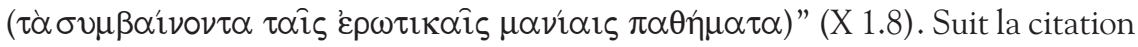
des 4 premières strophes de l'ode 31 .

Contentons-nous pour l'heure de relever l'éclairage particulier que le Pseudo-Longin donne à cette fureur ou transport érotique. Il faut ici revenir en arrière et rappeler que la citation de l'ode 31 voisine, dans le traité, avec des citations homériques qui la précèdent et qui mettent en valeur le souffle impétueux (oúpros $\sigma v v \varepsilon \mu \pi \nu \varepsilon i ̂)$ d'un Homère qui semble avoir composé ses épopées et surtout l'Iliade à la façon d'un héros livrant assaut sur le champ de bataille, un héros "plein de fureur": "Homère - dit-il - attise ( $\sigma v v \varepsilon \mu \pi \nu \varepsilon \hat{i})$ les combats de son souffle impétueux, et lui aussi est rempli de frénésie ( $\left.\mu \alpha^{\prime} \imath \varepsilon \tau \alpha \imath\right)$, comme on voit Arès brandir sa lance ou un feu dévastateur faire rage $\left(\mu \alpha^{\prime} \imath \eta \eta \alpha \imath\right)$ sur les montagnes..."54. Le commentaire se mêle et se confond ici à la citation de l'Iliade qui décrit l'assaut d'Hector. On remarquera avec N. Hertz $z^{55}$ que la distinction entre Homère et ses héros est comme effacée, cependant que la fureur guerrière est assimilée à la fureur de l'inspiration. L'emploi du verbe $\mu \alpha^{\prime} \mathbf{v}{ }^{\prime} \mu \alpha_{\mathrm{l}}$ ("je suis pris d'une ardeur furieuse, de délire" (s.v. DELG) prépare l'un de ces glissements qui fait l'intérêt du Пвpì v̌ oovs. Linspiration du poète homérique, comme rempli de fureur guerrière, prépare l'examen d'une deuxième forme de $\mu \alpha \nu$ í $\alpha$, érotique celle-ci, mais qui se confond, elle aussi, avec une forme de délire poétique. Les deux formes de fureurs sont différentes mais le parallélisme permet au Pseudo-Longin d'établir des similitudes qui associent Homère et Sappho: chacun expérimentant lui-même la $\mu \alpha v^{\prime} \alpha$ qu'il décrit et qui devient source ou plutôt force de son inspiration.

Linterprétation du Pseudo-Longin joue un rôle déterminant pour tout un courant qui assimile les sensations décrites dans l'ode 31 à des "pathêmata", des

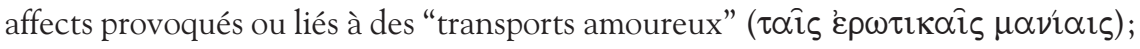
l'expression est d'autant plus difficile à traduire qu'elle est une transposition au

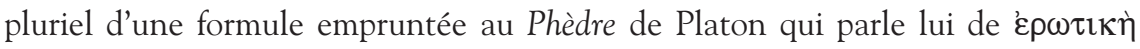
$\mu \alpha v_{i} \alpha^{56}$. L'emprunt est d'autant plus évident que le Pseudo-Longin cite plusieurs

54 Ps-Longin. De subl. IX 11; citant Il. XV.605-608.

55 Hertz, 1973, 292.

56 Cette désignation de $\pi \alpha \theta \eta \mu \alpha \tau \alpha$ lié à une fureur érotique ( $\tau \alpha i \varsigma \varsigma$ غ $\rho \omega \tau \imath \kappa \alpha i \varsigma \varsigma \mu \alpha \nu i \alpha \imath \varsigma$ )

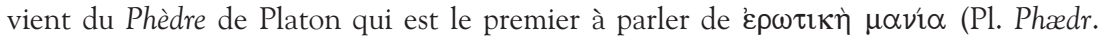
256d5; 265b5). Il faudrait, sans doute, examiner plus précisément tout ce que le traité 
fois Platon et reprend, notamment, le débat engagé dans le Phèdre sur le style rhétorique de Lysias. Linfluence de Platon sur le Пepì v́wovs est une donnée essentielle qui reste à étudier de près et qui n'est pas sans importance pour l'histoire de la réception de l'ode 31 - rappelons que le Phèdre contient une allusion très claire à l'ode 31 et que c'est le seul dialogue de Platon à citer le nom de Sappho, pour reconnaitre sa compétence à parler de l'amour ${ }^{57}$. Je me limiterai ici à indiquer quelques repères susceptibles de préparer un tel travail; il est cependant certain

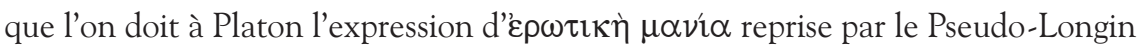
pour désigner l'origine des sensations $(\pi \alpha \theta \eta \mu \alpha \tau \alpha)^{58}$ décrites par Sappho.

Pour Platon, le Pseudo-Longin et toute une tradition dérivée de ces deux

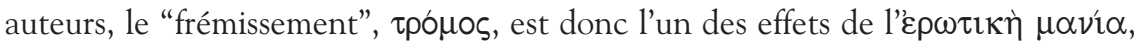
traduisons du "transport", du "délire" ou de la "fureur érotique", si l'on retient le

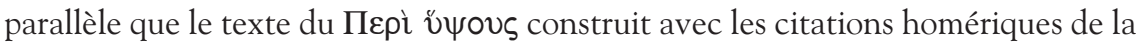
"fureur guerrière". Il est important ici de souligner un dernier parallélisme. Après avoir cité les quatre premières strophes de l'ode 31, le Pseudo-Longin loue encore Sappho pour sa façon de choisir et assembler les éléments les plus forts en un

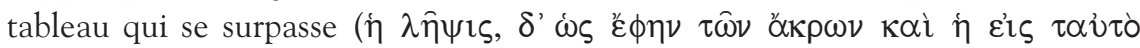

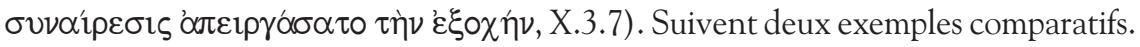
Le premier est une citation de l'auteur des Arimaspes, qui s'imagine avoir composé des vers terrifiants $(\delta \varepsilon \imath \nu \alpha ́)$, mais échoue à susciter la crainte $(\delta \varepsilon \dot{\varepsilon} \circ)$. Le second exemple, en contraste, est une citation de l'Iliade où Homère, contraignant les prépositions éloignées à s'unir (cf. $v \pi \varepsilon \dot{\varepsilon} \kappa)$ en dépit de leur nature ( $\sigma v v \alpha v \alpha \gamma \kappa \alpha ́ \sigma \alpha \varsigma$

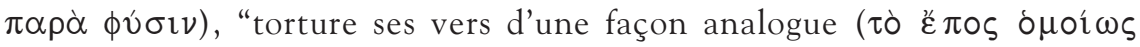
$\varepsilon \beta \alpha \sigma o ́ \omega l \sigma \varepsilon, X .7 .9)$ à la sensation ( $\pi \dot{\alpha} \theta 0 \varsigma$ ) évoquée; obtenant par "compression d'un mot" de reproduire une sensation qu'il semble imprimer dans la forme même

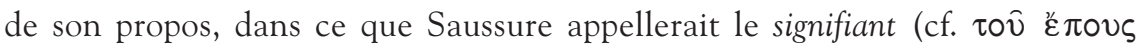

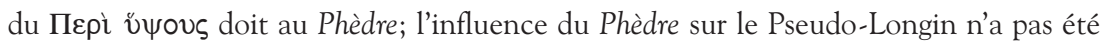
prise en compte par les interprètes de l'ode 31 - c'est une grave lacune; cf. par exemple Privitera, 1969b, 77 qui cherche l'origine ce "fureur érotique" chez Sappho elle-même: "E pero sintomatico che l'autore del Sublime questi segni li abbia definiti $\tau \dot{\alpha} \sigma v \mu \beta \alpha i$ -

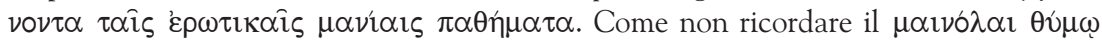
della preghiera [à Aphrodite, in Sapph. fr. 1.18 Voigt]"; Marcovich, 1972, 25 minimise aussi cet aspect.

57 Pl. Phædr. 235c3; pour l'allusion à l'ode 31 sur laquelle je reviens infra, cf. $251 \mathrm{a} 7$.

58 Remarquons aussi une occurrence de ce terme dans le Phèdre en 271b2, dans une phrase qui évoque les différents "genres" ( $\gamma \dot{\varepsilon} \nu \eta)$ de discours et d'âmes et leur $\pi \alpha \theta \eta \dot{\mu} \mu \tau \alpha$ réciproques pour réfléchir aux correspondances entre genres de discours et types d'âmes. 


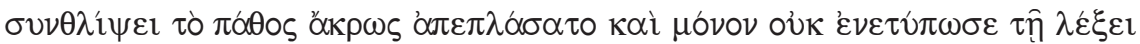

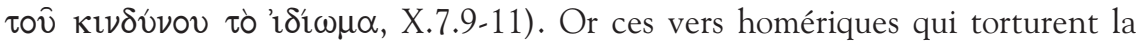
langue et portent en eux la marque du sentiment qu'ils décrivent ne sont autres que ceux d'une comparaison décrivant la peur. Hector est pareil à cette vague violente qui s'abat sur une nef: "et les marins, au fond d'eux-mêmes, frémissent,

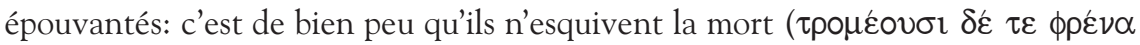

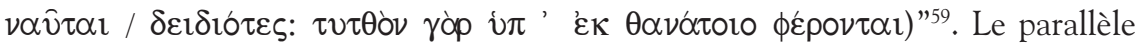
comparatif est à faire avec la fin de l'ode 31, telle que le Pseudo-Longin la découpe. L'impression d'être sur le point de mourir, le "frémissement" des marins ( $\tau \rho \circ \mu \varepsilon \dot{\varepsilon} \circ \sigma \mathrm{l})$ est provoqué ici par la peur qui envahit le "cœur" ( $\phi \rho \varepsilon ́ v \alpha)$. Par écho,

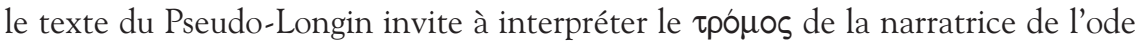
31 comme un frémissement de peur, lié à une forme de possession ou de fureur, un signe précédant et accompagnant la sensation d'une mort imminente. Indirectement, à travers le Пepì v̌wovs, ce sont Platon et Homère qui influencent notre appréhension de l'ode 31 .

\section{Le $\tau \rho o ́ \mu \circ \varsigma \varsigma$ comme expression d'extase chez Boileau et Madame Dacier}

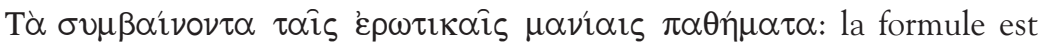
puissante; pour beaucoup, elle définit, à la façon d'un titre, le sujet de l'ode 31 du moins des strophes que le Pseudo-Longin a choisi de citer. Quant à la méthode de composition, elle est identifiée comme une alternance de mouvements opposés et complémentaires. Sappho est exemplaire dans cet art de la $\lambda \hat{\eta} \psi r \varsigma$ et de la

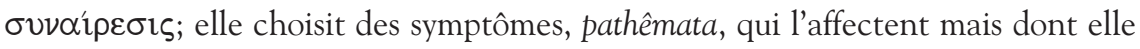
se détache comme "comme autant de choses qui lui sont étrangères et qui se

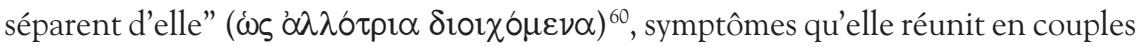
de contraires: $\kappa \alpha \theta^{\prime}$ ' $\alpha \tau \varepsilon v \alpha \nu \tau \tau \omega \sigma \varepsilon \imath \varsigma$. Linterprétation du Pseudo-Longin - qui sera reprise et développée notamment par G. A. Privitera - est largement fondée sur ce dédoublement des sensations contraires. Sappho "est transie de froid et elle brûle;

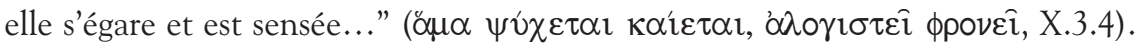
Remarquons qu'une telle lecture implique la présence de l'adjectif $\psi v \chi$ pós pour

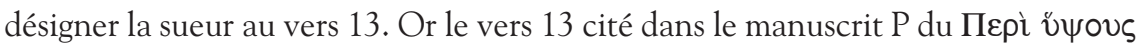
est métriquement impossible et par ailleurs le maintien du masculin $\psi v \chi$ xós fait problème si l'on rappelle, d'après un témoignage qui remonte sans doute à Hérodien,

59 Il. XV.627-8. (trad. P. Mazon).

${ }^{60}$ Expression que Privitera, 1969a, 31, traduit par "andati via tutti come non suoi". 
qu'en éolien " $\delta \rho \omega \varsigma$ est féminin ${ }^{61}$. Plusieurs éditeurs (dont Voigt) ont choisi de

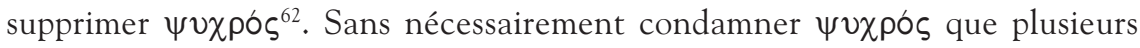
imitations retiennent ${ }^{63}$, il ne semble pas cependant que l'opposition des sensations contraires soit une structure fondamentale de l'ode 31. Elle est plutôt une projection du Pseudo-Longin qui veut reconnaître à Sappho un art qu'il revendique pour luimême: celui d'unir en un tout des éléments distincts mais choisis.

Malheureusement la suite du texte fait problème et, dans la liste des pathêmata contraires évoqués par l'ode 31 , certains éditeurs ont placé plusieurs

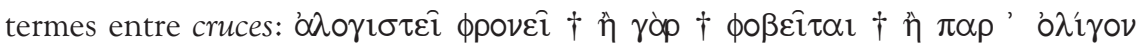

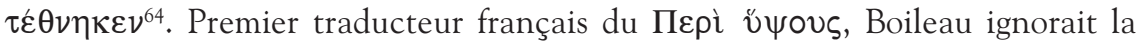
possibilité d'un róp explicatif et allongeait la liste des mouvements contraires qu'il reconnaît, à la suite du Pseudo-Longin, comme la marque du style de Sappho: "Voyez de combien de mouvements contraires elle est agitée. Elle gele, elle brûle, elle est folle, elle est sage; ou elle est entierement hors d'elle-mesme, ou elle va mourir [...] Son ame est un rendez-vous de toutes les passions" ${ }^{65}$. Dans son commentaire à ce passage, Boileau justifie rapidement sa traduction, pour le moins

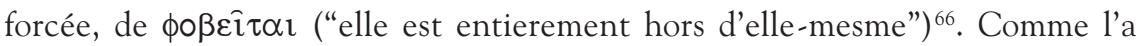
remarqué, Joan DeJean, la traduction de Boileau affaiblit l'ode 31; il modifie notamment l'expression "plus verte que l'herbe" par "pâle"; il élimine aussi l'expression "sueur froide". Concentrant son attention sur le thème de la $\mu \alpha v^{\prime} \alpha$, il transforme "la peur qu'inspire la puissance de l'amour en portrait d'une femme qui s'abandonne à la folie" ${ }^{67}$. Boileau interprète à contresens la $\mu \alpha v^{\prime} \alpha$ du PseudoLongin; il substitue à une force active, un statut passif. Dans le Пepì v́พovs, la $\mu \alpha v_{i} \alpha$ est tout à la fois la force dévastatrice qui semble défaire le corps de la narratrice et la force qui lui permet de se reconstruire, dans son poème, en évoquant

${ }^{61}$ Epim. An. Ox. I 208.13-15 Cramer.

62 Privitera, 1969b, 41 apporte des arguments en faveur du maintien de $\psi v$ vó́s; contra Di Benedetto 1985, 151-4 et Tzamali, 1996, 183-6. Lanalyse de Neri \& Citti, 2003, qui invite $(58, \mathrm{n} .26)$ à nuancer l'importance du témoignage d'Hérodien, apporte une contribution essentielle sur la complexité du débat et sur les raisons de maintenir uvxpós (57).

63 Pl. Phædr. et Theoc. II.105-11.

${ }^{64}$ Je cite ici le texte édité par Russell, 1982.

65 Traité du sublime, trad. Boileau in Adam \& Escal, 1979, 357.

66 Ibid. p. 417.

67 Dejean, 1994, 79. 
"comme autant de choses qui lui sont étrangères et qui se séparent d'elle" les parties de son corps touchées par la $\mu \alpha v^{\prime} \alpha$. Lart de Sappho, d'après le PseudoLongin, est de savoir transformer cette fureur en "énergie qui constitue le poème", de savoir transformer en inspiration poétique la violence de la passion amoureuse ${ }^{68}$.

Répondant à Boileau, Madame Dacier rend son sens premier au verbe $\phi \circ \beta \varepsilon i \tau \alpha$, mais pour voir dans ce mouvement de crainte une sorte de frisson extatique. Dans son édition des Odes d'Anacréon et de Sappho, elle ouvre une note

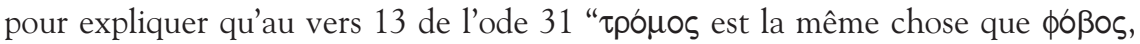

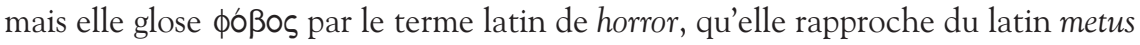
pour conclure de façon paradoxale et sous l'influence de Boileau qu'il s'agit d'"un certain tremblement qui vient de fureur, tels que sont les transports que sentent ceux qui sont saisis de l'esprit d'un Dieu". Et, d'ajouter, en comparant ce $\tau \rho{ }^{\prime} \mu \varsigma$

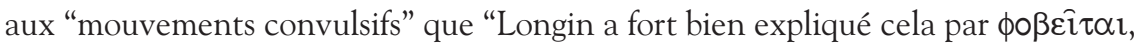
trepidat, elle est éperduë, elle est hors d'elle-mesme" ${ }^{69}$. Madame Dacier achoppe sur la

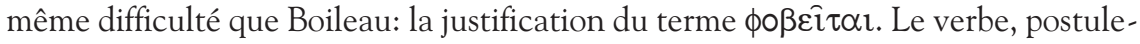

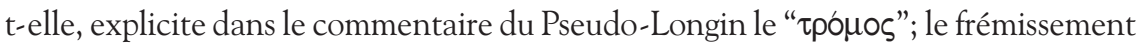
est signe d'une extase. Il s'agit d'amour pour Boileau, d'amitié pour Madame Dacier, mais dans les deux cas, la crainte est niée pour être assimilée à une forme d'égarement, propre à une hypersensibilité féminine. On sait que Racine s'est inspiré de l'ode 31 de Sappho et de la traduction de Boileau pour composer les vers peutêtre les plus célèbres de sa Phèdre (1677) quand son héroïne, déchirée par sa passion, confie:

Je le vis, je rougis, je pâlis à sa vue;

Un trouble s'éleva dans mon âme éperdue;

mes yeux ne voyaient plus, je ne pouvais parler;

je sentis tout mon corps et transir et brûler.

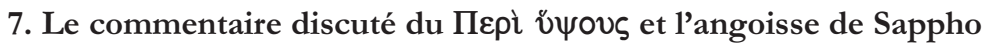

Mais revenons au commentaire que le Pseudo-Longin donne de l'ode 31 et à la ligne qui pose problème. Récusant l'idée d'une difficulté syntaxique ou

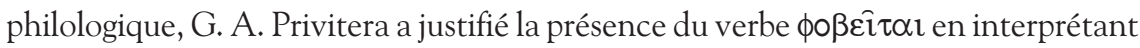

68 Hertz, 1973, 295.

69 Les poésies d'Anacréon et de Sapho, traduites de grec en françois, avec des remarques, par Madame Dacier, Paris, chez D. Thierry \& C. Barbin, 425. 


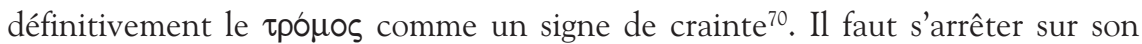
interprétation qui aura son influence. Loin de vouloir transformer le texte ou supprimer $\phi o \beta \varepsilon i \tau \alpha \mathbf{l}^{71}$, retraçant les grandes lignes du débat philologique déjà rappelées par Jahn et Valens (1910), Privitera repousse l'argument d'une maladresse dans l'emploi de î rò̀ avec hiatus après $\phi \rho \circ v \varepsilon^{72}{ }^{72}$; il n'est pas plus convaincu par l'explication de la junctura malheureuse ( $\hat{\eta}$ ròp ... $\hat{\eta}$...) dont d'autres exemples sont clairement attestés ${ }^{73}$. La difficulté de l'expression réside, note-t-il, dans le sens apporté par cette explication et dans l'équivalence qu'il faut établir entre

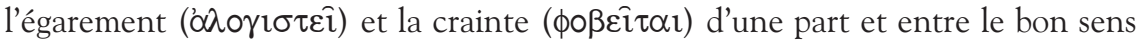

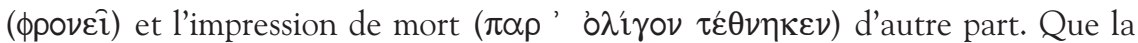
crainte puisse ravir la raison, Privitera en trouve un exemple dans l'Eloge d'Hélène de Gorgias $^{74}$. Quant à la deuxième équivalence, l'explication de Privitera est

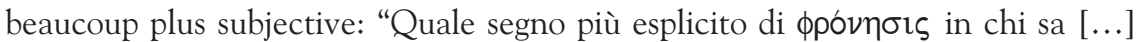
non di essere ma di sembrare à se stessa, non morta ma quasi morta?"75 Soulignant

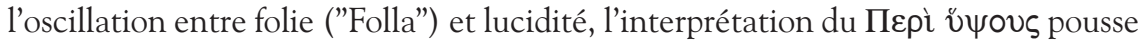
Privitera à donner un sens fort au frémissement ( $\tau \rho \circ \operatorname{\mu o\zeta })$ : "il tremito che collie tutta la persona è il segno più diretto ed esplicito di quelle sbigottimento che colpisce

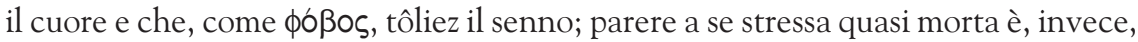
un segno caractéristique di chi vede ludicamente la proprio condizione"76.

Dans un second article, publié la même année, Privitera poursuit son analyse en l'ouvrant à la composition même de l'ode 31 et en s'intéressant à l'articulation des différents symptômes. Plusieurs références homériques lui permettent de

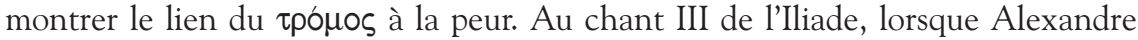

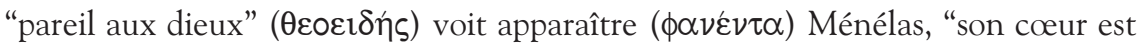

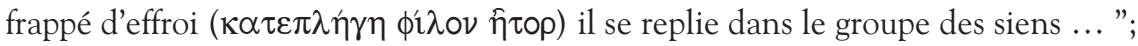
pareil à un homme qui a vu un serpent dans les gorges des montagnes, "ses membres

70 Privitera, 1969b, 32.

71 Comme Weiske, 1820 (paperback chez Nabu Press en 2010) et plus récemment encore Mazzucchi, 1992, 188, qui proposent tous deux de supprimer toute l'expression

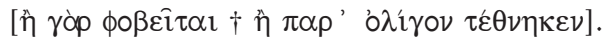

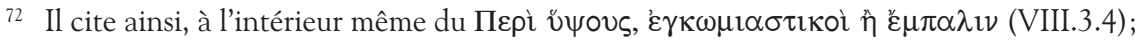

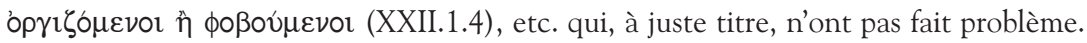

73 Privitera, 1969b, 31-2 qui cite Hdt. VII.51.2; Pl. Ap. 40c, etc.

74 Gorg. Hel. 11.17.

75 Privitera, 1969a, 33.

76 Privitera, 1969a, 35. 


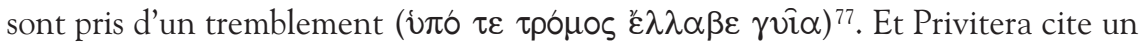
deuxième passage que l'on peut citer plus longuement qu'il ne le fait, vu son intérêt. Au chant $\mathrm{X}$ de l'Iliade, la nuit n'apporte pas de répit à Agamemnon mais "dans sa poitrine, il sent se presser les sanglots; ils montent du fond de son cœur; toutes ses

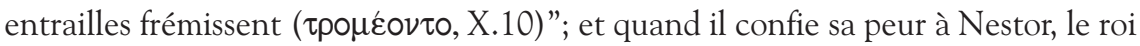
achéen se fait plus explicite: "J'ai terriblement peur pour les Daniens; mon âme est sans repos et je me sens en plein égarement ( $(\alpha \lambda \alpha \lambda \hat{v} \kappa \tau \eta \mu \alpha \imath)$. Le coeur me bondit

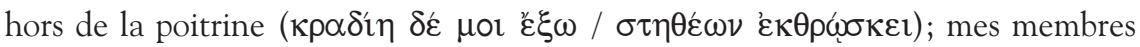
brillants sont là qui tremblent ( $\tau \rho \rho \mu \varepsilon \varepsilon 1)$ sous moi." (X.93-5).

Mais l'analyse de Privitera ne s'arrête pas là. Regroupant les symptômes par couple dans un schéma qui évoque une structure annulaire ou géométrique ${ }^{78}$, il

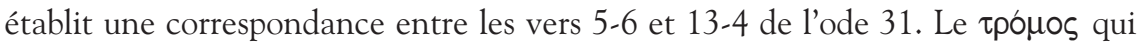
secoue la narratrice est ainsi la donné symétrique de l'émotion qui affole son cœur:

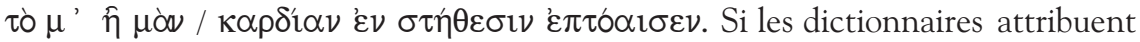
au verbe $\pi \tau 0 \varepsilon \omega$ ( $\pi \tau 01 \varepsilon \omega)$ le sens premier de "terrify, scare" (LSJ), "frapper d'effroi, terrifier" (Bailly), "ängstigen" ou, au passif, "erschreckt, leidenschaftlich erregt werden" (Frisk), Privitera invite à nuancer cette valeur. Chez Homère, le verbe, attesté deux fois, est certes employé avec cette connotation, lorsqu'Ulysse menace les servantes et lorsqu'Athéna brandit son égide devant les prétendants (Od. XVIIII 340 et XXII 298); mais d'autres emplois de $\pi \tau \circ \varepsilon ́ \omega$ chez Hésiode, Apollonios de Rhodes et Anacréon invitent à lui donner un sens plus général: "Il verbo è un termine tecnico e indica un'azione che si esercita su taluni organi (le фpદ́ves o la $\kappa \alpha \rho \delta i \alpha$, come in quest'ode) in cui hanno sede le facoltà psichiche: quest'azione è di disturbo e tale da impedire il coordinamento delle facoltà verso un fine" ${ }^{\text {" }}$. Les sens ou le cœur peuvent être agités, troublés et dérangés par divers facteurs. Pour Privitera, l'emploi du verbe $\pi \tau 0 \varepsilon ́ \omega$ permet à Sappho de jouer sur la multiplicité de ses valeurs: la peur comme chez Homère, la stupeur comme chez Hésiode, la folie comme chez Euripide, ou l'amour comme chez Anacréon ou Apollonios ${ }^{80}$. La narratrice de l'ode 31 éprouverait les quatre sensations à la fois. Mais l'art de Sappho est dans "la tension stylistique" qu'elle établit entre ces symptômes, pathêmata,

77 Il. III.34 et la note 34 de Privitera, 1969 Voir aussi pour le verbe: Il. X.390.

78 Privitera, 1969a, 61: "Si potrebbe concepire ordine piu rigorosamente geometrico?"

79 Hes. Erg. 447; Ap. Rh. I 1232; Anacr. fr. 346 1.12 Page. Pour la citation, Privitera, 1969a, 58.

80 Privitera, 1969b, 59. 
qu'elle associe ou oppose en une structure très travaillée. Il faut alors combiner l'affolement du cœur et le tremblement du corps, l'aphasie et la voix brisée, le feu et la sueur froide, la vue brouillée et l'ouïe bourdonnante, la pâleur et le sentiment de mort, pour saisir la complexité d'une émotion qui renvoie aux connotations multiples attachées à ces symptômes par les autres poètes. Le verbe $\varepsilon \pi \tau o ́ \alpha \iota \sigma \varepsilon v$ (de $\pi \tau 0 \varepsilon \dot{c} \omega)$ est alors, au vers 6 , la clé de voûte ("chiave di volta") de cette construction $^{81}$, mais sa position qui fait de lui la donnée symétrique du tremblement, ses connotations homériques, font que, dans cette ode - qui dit la stupeur, la peur, la douleur et l'amour - la peur est prioritaire, tandis que l'amour reste moins explicite et "ne devait émerger comme dénominateur commun qu'après la lecture de la partie perdue et conclusive de l'ode" 82 .

Indépendamment de l'étude de Privitera et avec une méthode et des concepts différents, G. Devereux développe une analyse de l'ode 31 qui interprète les symptômes comme une véritable crise d'anxiété homosexuelle. Insistant, comme Page, sur la présence d'un homme réel assis face à la femme au rire désirable, Devereux souligne la frustration de la narratrice identifiée à Sappho: "What does this man - and indeed any man - have that Sappho does not have? What can a man offer to a girl that Sappho cannot offer?"83. La réponse de Devereux est un constat psychanalytique sur le "female castration complex" ressenti par "the masculine lesbian" et qui provoque la crise d'anxiété. Il rappelle, dès le début de son étude, que la peur, "fear", ne doit pas être confondue avec l'anxiété; mais il n'en reste pas moins que sa position peut-être vue comme un développement de l'observation de Privitera. Si Devereux invite à ne pas confondre anxiété et peur, le philologue italien ne s'embarrasse pas de cette distinction: il est vrai que pour l'helléniste la nuance est plus délicate à établir; le vocabulaire grec ne connaît pas de concept propre à signifier l'anxiétée 84 . Dans sa conclusion, Privitera évoque ainsi

81 Privitera, 1969b, 59.

82 Privitera, 1969a, 67-8: "Letti controluce i vari disturbi rivelano legami secondari che si intersecano tra coppia e coppia in una trama fitta e variegata di motivi: lo stupore, la paura, il dolore, l'amore, la morte. Il più insistito è la paura. Il meno esplicito è l'amore, che doveva emergere come denominatore comune soltanto dopo la lettura della parte perduta e risolutiva dell'ode." Voir aussi Lasserre, 1989, 152, pour qui Privitera, 1969b insiste "avec raison sur la prédominance de la peur dans cette ode". Cf. aussi Calame, $1977,430$.

83 Devereux, 1970, 22.

84 Ainsi Calame, 1977, 430: "La crise de Sappho est vraisemblablement provoquée par la vue de son rival masculin auquel, en tant que femme, la poétesse ne peut se substituer 
une Sappho "en proie à l'anxiété" qui, dans l'ode 31, a voulu expliquer "l'improvviso terrore" qui l'a saisi à la vue de la femme désirée: "Come significare questo aspetto singolarismo del suo sentimento, questa condizione di innamorata che trema e le ragioni di questo tremare, che certo non è tipico di qualunque specie di tremore? Come dire che l'ansia la paralizza in misura quasi mortale?" 85 Le terme d'anxiété ("ansia") est ici prononcé par Privitera, qui rejoint Devereux dans son diagnostic ${ }^{86}$, mais explique psychologiquement ce que le psychanalyste expliquait en termes psychanalytiques. Au complexe lesbien de "female castration" avancé par Devereux répond chez Privitera une Sappho qui aime sans espoir de retour: "Dare un corpo ai fantasmi del proprio timore senza ridurli a dati obiettivi e irreversibili; esprimere questo stato angoscioso di sospensione tra immaginazione e realtà; descrivere $\mathrm{i}$ disturbi della paura e significare i segni dell'amore...". Le déchirement entre des passions contradictoires est ici la source d'une force poétique. Le génie de Sappho est, pour Privitera, d'avoir su "entrelacer" ("intrecciare") des données autorisant deux lectures: "quella della paura con Lucrezio; quella dell'amore con Teocrito e gli altri" ${ }^{87}$. Nous voilà ramenés à la double lecture des Anciens, mais pour aboutir à une tentative de synthèse où l'anxiété domine.

Tout récemment encore, le débat est relancé avec force par Franco Ferrari qui s'appuie sur les données statistiques publiées par l'American Psychiatric Association pour établir un lien entre les symptômes décrits dans l'ode 31 et ceux manifestés dans une crise de panique. Pour lui, c'est évident, le $\tau$ pó $\mu \circ \varsigma$, associé aux autres symptômes, est signe d'une crise de panique qui perturbe le désir amoureux. Et dans le monde contemporain, ces crises sont courantes: elles toucheraient entre $3 \%$ et $4 \%$ de la population de la planète, "senza sensibili differenze statistiche legate a diverse condizioni sociali o a diverse aree politiche e culturali" ${ }^{8}$. Significativement la différence sexuelle n'est pas relevée. Faut-il supposer qu'elle est sans pertinence? F. Ferrari en tout cas s'écarte, sur ce point, de

auprès de la jeune fille aimée (Devereux, 1970, 22s.). G. A. Privitera (1969b) l'a bien vu quand il dit que les symptômes énumérés par Sappho sont les signes de la terreur qu'elle éprouve en pensant que son amour pour la jeune fille est sans espoir et ne sera pas payé de retour".

85 Privitera, 1969b, 78.

86 Contre cette lecture de Devereux, cf. Marcovich, 1972, qui invite à prendre en compte l'influence d'une tradition poétique sur une expérience personnelle (cf. 26).

87 Privitera, 1969b, 68.

${ }_{88}$ Ferrari, 2007, 161 et 162, n. 2, pour sa critique de Devereux, 1970. 
l'analyse de G. Devereux et préfère reformuler le problème en recourant aux notions de la médecine hippocratique.

Tremblement d'une fièvre ou d'un malaise amoureux, signe d'un état extatique d'égarement, marque d'un délire amoureux qui devient force créatrice,

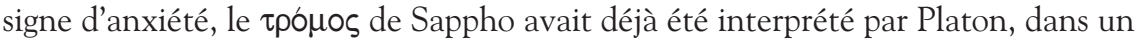
texte qui a influencé le Pseudo-Longin, mais je n'aborderai ce point en conclusion que pour annoncer une enquête à suivre.

\section{Platon et la resémantisation du frémissement de Sappho}

Comme Gorgias dans son Eloge d'Hélène, Platon confronte dans le Phèdre la force d'Eros ${ }^{89}$ et le pouvoir du lógos. Cette confrontation est aussi une compétition rhétorique qui voit Socrate développer une riche réflexion sur les formes de possession et de "folie", $\mu \alpha v^{\prime} \alpha$, en lien avec le divin et susceptibles d'élever l'âme. Mais avant de parler de l'âme, Socrate a écouté le discours de Lysias sur l'amour. Le cadre du dialogue est privilégié pour une rencontre avec Eros. Les deux compagnons se sont assis - puis Socrate s'est étendu ${ }^{90}$ - sur un carré d'herbe tendre, "sous une brise modérée" ${ }^{1}$, à l'ombre d'un haut platane et d'un gattilier dont les fleurs ont un parfum qui embaume tout le lieu. Une source fraiche - "la plus charmante", $\chi \alpha \rho ı \varepsilon \sigma \tau \alpha ́ \tau \eta ~(230 b 5)$ - coule sous le gattilier. Les bords du ruisseau

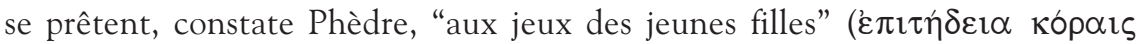
$\left.\pi \alpha^{\prime} \zeta \zeta \varepsilon t v, 229 \mathrm{~b} 8\right)$. L'atmosphère invite à une rêverie sur Eros ${ }^{92}$ et l'on ne sera pas surpris d'entendre bientôt le nom de Sappho. Alors que Phèdre demande à Socrate à qui il pense en affirmant qu'il existe sur l'amour de meilleurs discours que celui de Lysias, le philosophe prononce le nom de Sappho, sans être plus précis, ni citer de poème en particulier. On pourrait oublier cette allusion, si le deuxième discours de Socrate ne posait pas directement la question de l'effet de la beauté sur l'âme. Immortelle, l'âme retrouve dans les beaux visages qu'elle peut voir durant sa vie terrestre comme un reflet de la beauté véritable (245d5-6); elle est alors sous l'effet

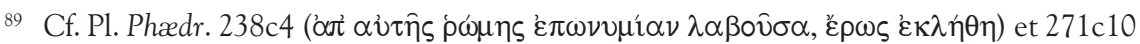
( $\lambda$ ó

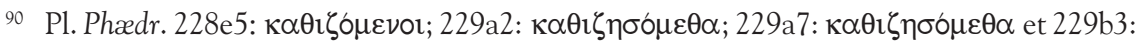

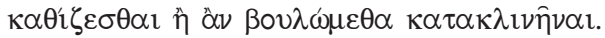

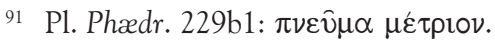

92 Comme l'ont bien souligné Calame, 1996, 173-5 et 206 et Pender, 2007b, 3-8 qui aborde parfaitement le rapport de Platon avec Sappho; Voir aussi Pender, 2007a. 
de la plus belle forme de "possession" ou de "folie" qui puisse être. C'est l'expérience de qui contemple un beau garçon. Il n'est pas sûr que le spectacle suffise à chaque fois à réveiller le souvenir de la beauté vraie, parfois à peine entrevue quand l'âme, avant sa réincarnation, cheminait dans le ciel pour être initiée à cette contemplation de l'être en soi. Mais, si la mémoire se met en branle, l'âme est prise d'un délire, "projetée hors d'elle-même, ne se possédant plus" (250a6-7). Lamour du beau est ici une expérience métaphysique, elle relève de la $\mu \alpha v^{\prime} \alpha$ et sera d'autant plus forte qu'elle touchera un amant fraichement initié, encore marqué par la contemplation du beau en soi. Celui-là, "quand il voit un visage d'aspect divin $(\theta \varepsilon \circ \varepsilon 1 \delta \varepsilon \xi \varsigma)$, qui est une heureuse imitation de la beauté [...], il commence par

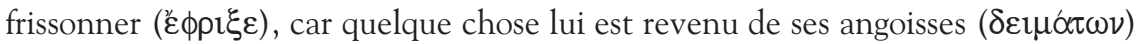
de jadis. Puis, il tourne son regard vers cet objet, il le vénère à l'égal d'un dieu et, s'il ne craignait de passer pour complètement fou, il offrirait au jeune garçon des sacrifices comme à la statue d'un dieu, comme à un dieu"'33.

Depuis Homère, la poésie n'a cessé de réinventer les scènes d'admiration et de contemplation, les face-à-face visuels. L'lliade se termine sur le face-à-face d'Achille et de Priam et sur les mots d'Hélène qui dit l'horreur de ceux qui la regardent. Sappho a chanté la force des sensations qui la paralysent face à une femme au sourire inaccessible. Platon décrit le face-à-face avec un visage qui reflète la beauté en soi et inspire la plus belle forme de $\mu \alpha v^{\prime} \alpha$ : "Or, en l'apercevant, il change à la suite du frisson ( $\left.\varepsilon \kappa \tau \eta \hat{\zeta} \varsigma \phi \rho^{\prime} \kappa \eta \varsigma\right)$; il se couvre de sueur; il éprouve une

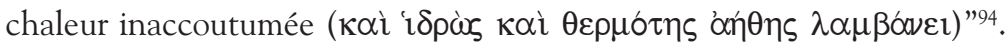

Les commentateurs du Phèdre ne s'y sont pas trompés: Platon se souvient bien ici des symptômes de l'ode 31 de Sappho ${ }^{95}$. Mais au face-à-face féminin évoqué

93 Pl. Phædr. 251a2-7.

94 Pl. Phædr. 251a7-b1.

95 Fortenbaugh, 1966, 108-9, remarque à juste titre que les mentions des noms d'Anacréon et Sappho en 253c3 "are note general reference to lyric love poets" mais "are introduced to alert the reader that the poem of Sappho and Anacreon will play a role in Socrates' subsequent speeches"; s'il cite plusieurs échos pertinents, y compris pour Anacréon, Fortenbaugh ne mentionne pas l'écho de 251a7-b1 avec Sapph. fr. 16.13-4 Voigt; écho en revanche noté par De Vries, 1969, 154; Heitsch, 1993, 36; Reale, 1998, 224. Ferrari, 2007, 161 souligne le rapprochement mais sans parler d'allusion. Voir également le commentaire de Ferrari, G. R. F., 1988; 153-7; duBois, 1995 85-6 et Foley, 1998, 40-4. Mais la question des citations de Sappho et Anacréon dans le Phèdre a surtout intéressée Pender (2007a et b) qui en donne un traitement riche. 
par Sappho, il substitue un face-à-face masculin. Et surtout, il a substitué au terme

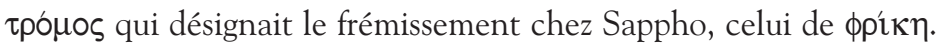

A ce point, c'est une nouvelle étude qu'il faut écrire qui devra répondre à deux questions. Quelle fonction a dans ce passage clé du Phèdre une allusion à Sappho (notons que c'est la première allusion connue de la prose à l'ode 31 ) et pourquoi, évoquant le "frémissement", Platon a-t-il substitué au terme attendu

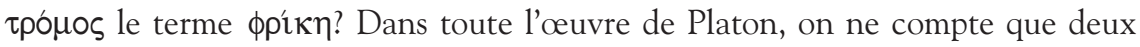
occurrences de ce terme. La seconde est dans la République dans un passage où Platon décrit le "frémissement d'horreur" qui saisit les gardiens lorsqu'ils entendent les poètes réciter "ces noms terribles et effrayants ( $\phi \circ \beta \varepsilon p \alpha)$, tels Cocyte, Styx, mânes, spectres, et tous les noms du même genre qui font frissonner ( $\phi \rho i ́ \tau \tau \varepsilon \imath v)$ ceux qui les entendent" ${ }^{\prime \prime}$. Surtout, Socrate craint qu'un tel "frisson" ne les échauffe

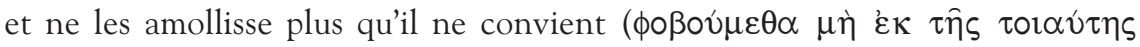

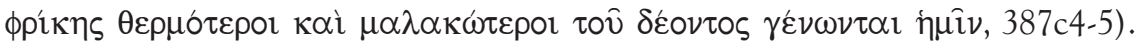
Ce frisson d'horreur, proche de celui que suscite Hélène dans l'lliade, est à l'opposé de celui qui saisit l'âme admirant le beau dans le Phèdre. Frémissement d'horreur devant la mort ou frémissement lié à un transport érotique ( $\left.\varepsilon \rho \omega \tau \imath \kappa \eta े ~ \mu \alpha v^{\prime} \alpha\right)$ ? Une histoire du "frisson" en Grèce ancienne devra consacrer un chapitre à Platon.

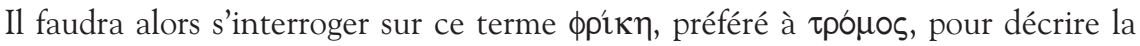
sensation de l'âme à la vue d'un visage lui rappelant la beauté en soi. Pourquoi, alors même qu'il se souvient de Sappho, Platon emploie-t-il un mot rare et connoté qui renvoie à l'effet que provoque Hélène dans l'Iliade? Une Hélène devant qui tous frissonnent ( $\left.\pi \dot{\alpha} \nu \tau \varepsilon \varsigma \zeta \delta \varepsilon \mu \varepsilon \pi \varepsilon \phi \rho^{\prime} \kappa \alpha \sigma \iota v\right)$, sans que le poète ne précise - parce que cela semble évident $-s^{\prime}$ il s'agit d'un frisson d'horreur ou d'émerveillement ${ }^{97}$.

\section{RENSEIGNEMENTS ET REMERCIEMENTS}

Cette étude prolonge un exposé présenté dans le cadre du Programa de Pós-Graduação em Letras Clássicas de l'Université de São Paulo, le 1er décembre

96 Pl. Resp. 387b8-c5.

97 Platon invite à reprendre le débat sur l'image d'Hélène chez Sappho (en pensant au fr. 16 mais pas seulement); question riche d'une importante bibliographie, signalons duBois 1978 (repris dans Greene, 1996a, 79-88) et 1995; et, dans une optique qui critique la précédente, Calame, 1987 repris et remanié in Calame, 2005, 107-130. Voir aussi Pellicia, 1992. 
2008. Je remercie en particulier Paula da Cunha Corrêa, Mary de Camargo Neves Lafer et Marcos Martinho dos Santos pour leurs conseils et pour les traductions en portugais qu'ils m'ont procurées de l'ode 31 .

\section{Bibliographie}

Acosta-Hugues, B., 2010. Arion's Lyre. Archaic Lyric into Hellenistic Poetry, Princeton: Princeton University.

Adam, A. \& F. Escal, 1979. Boileau. Oeuvres complètes, Paris: Gallimard.

Bouvier, D., 2009. "Peut-on traduire une émotion érotique? Lexemple des traductions françaises de l'ode 31 de Sappho". Social Science Information, 48, 465-85.

Brunet, P., 1998. L'égal des dieux: cent versions d'un poème de Sappho, Paris: Allia.

Calame, C., 1977. Les chœurs de jeunes filles en Grèce archaïque, Rome: Edizioni dell'Ateneo \& Bizzarri.

Calame, C., 1987. "Sappho et Hélène. Le mythe comme argumentation narative et parabolique", in Parole-figure-parabole. Recherches autour du discours parabolique, ed. Delorme, J. Lyon: Presses universitaires de Lyon.

Calame, C., 1996. L'Eros dans la Grèce antique, Paris: Belin.

Calame, C., 2005. Masques d'autorité : fiction et pragmatique dans la poétique grecque antique, Paris: Les Belles Lettres.

De VRIes, G. J., 1969. A commentary on the Phaedrus of Plato, Amsterdam: A.M. Hakkert.

Degani, E. \& G. Burzacchini, 1977. Lirici greci antologia, Firenze: La nuova Italia.

Degani, E. \& G. Burzacchini (eds.), (2005). Lirici greci : antologia (con aggiomamento bibliografico a cura di Massimo Magnani), Bologna: Pàtron.

DeJeAn, J., 1989. Fictions of Sappho (1546-1937), Chicago - London: Unversity Press of Chicago.

DeJean, J., 1994. Sapho. Les fictions du désir 1546-1937, Paris: Hachette.

Devereux, G., 1970. "The Nature of Sappho's Seizure in fr. 31 LP as Evidence for Her Inversion”. Classical Quarterly, NS 20, 17-31.

Di Benedetto, V., 1985. "Intorno al linguaggio erotico di Saffo". Hermes, 113, 145 9. 
DuBors, P., 1978. "Sappho and Helen". Arethusa, 11, 89-99.

DuBors, P., 1995. Sappho is burning, Chicago - London: University of Chicago Press.

FERRARI, F., 1986. "Formule saffiche e formule omeriche". Annali della Scuola Normale Superiore di Pisa (III), 16, 441-7.

Ferrari, F., 2007. Una mitra per Kleis : Saffo e il suo pubblico, Pisa: Giardini.

Ferrari, G. R. F., 1988. Listening to the cicadas : a study of Plato's "Phaedrus", Cambridge ; New York etc.: Cambridge University Press.

Foley, H. P., 1998. “"The mother of the Argument”: Eros ans the Body in Sappho and Plato's Phaedrus", in Parchments of gender, ed. WyKE, M. Oxford - New York: Clarendon Press - Oxford University Press, 39-70.

Fortenbaugh, W. W., 1966. "Plato's Phaedrus 235c3". Classical Philology, 61(108-109).

Fowler, R., 1986. "Leah Rissman: Love as War: Homeric Allusion in the Poetry of Sappho (review)". Classical Review, NS, 36, 301-2.

Greene, E. (ed.) (1996a). Reading Sappho. Contemporary Approaches, Berkeley Los Angeles - London.

Greene, E. (ed.) (1996b). Re-Reading Sappho. Reception and Transmission, Berkeley - Los Angeles - London.

Hainsworth, B., 1993. The Iliad: a Commentary, Books 9-12, Cambridge: Cambridge University Press.

Heitsch, E., 1993. Phaidros. Platon Werke. Übersetzung und Kommentar, Göttingen: Vandenhoeck \& Ruprecht.

Hertz, N., 1973. "Lecture de Longin". Poétique, 4, 292-319.

Hutchinson, G. O., 2001. Greek lyric poetry a commentary on selected larger pieces, Oxford: Oxford University Press.

Iriarte, A., 2007. "Chanter, enchanter en Grèce ancienne. À propos de Sappho, femme poète et dixième Muse". Clio. Histoire, femmes et sociétés, 25, 27-43.

Jahn, O., J. Vahlen \& H.-D. Blume, 1967 (1910). Dionysii vel Longini De sublimitate libellus, Stutgardiae: in aedibus B.G. Teubneri.

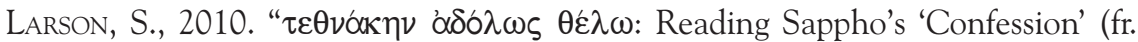
94) through Penelope". Mnemosyne, 63, 175-202.

Lasserre, F., 1989. Sappho. Une autre lecture, Padoue: Antenore.

LefKowitz, M. R., 1996. "Critical Stereotypes and the Poetry of Sappho", in Reading Sappho. Contemporary Approaches, ed. Greene, E. Berkeley "Los Angeles London, 26-34. 
Lightfoot, J. L., 2003. Lucian. On the Syrian Goddess. Introduction. Translation $\mathbb{E}$ Commentary, Oxford: Oxford University Press.

Marcovich, M., 1972. "Sappho Fr. 31: Anxiety Attack or Love Declaration?". The Classical Quarterly, NS 22, 19-32.

Mattioli, E., 1988. Interpretazioni dello Pseudo-Longino, Modena: Mucchi.

Mazzucchi, C. M., 1992. Dionisio Longino. Del sublime, Milano: Vita e Pensiero.

Mesk, J., 1913. "Antiochos und Stratonike". Rheinisches Museum, 68, 399-4.

Michel, A., 1976. "Rhétorique et poétique: la théorie du sublime de Platon aux modernes". Revue des Etudes Latines, 54, 278-307.

Most, G. W., 1996. "Reflecting Sappho", in Re-Reading Sappho. Reception and Transmission, ed. GreEne, E. Berkeley - Los Angeles - London, 11-35.

Neri, C. \& F. CitTi, 2003. "Sudore freddo e tremore". Eikasmos, 16, 52-62.

PAGE, D. L. (ed.) (1955). Sappho and Alcaeus : an introduction to the study of ancient Lesbian poetry, Oxford: at the Clarendon Press.

Pellicia, H., 1992. "Sappho 16, Gorgias' Helen, and the preface to Herodotus' Histories”. Yale Classical Studies, 29, 63-84.

Pender, E. E., 2007a. "Poetic allusion in Plato's Timaeus and Phaedrus". Göttinger Forum fur Altertumswissenschaft, 10, 21-57.

Pender, E. E., 2007b. "Sappho and Anacreon in Plato's Phaedrus". Leeds International Classical Studies, 6.4, 1-57 (http://www.leeds.ac.uk/classics/lics/2007/ 200704.pdf).

Prins, Y., 1996. "Sappho's Afterlife in Translation", in Reading Sappho. Contemporary Approaches, ed. Greene, E. Berkeley - Los Angeles - London, 36-67.

Privitera, G. A., 1969a. "Il commento del ðåñv Uøĩõò al fr. 31 L.P. di Saffo". Quaderni Urbinati di Cultura classica, 7, 26-35.

Privitera, G. A., 1969b. "Ambiguità antitesi analogia nel fr. 31 L.P. di Saffo". Quaderni Urbinati di Cultura classica, 8, 37-80.

Ragusa, G., 2005. Fragmentos de uma deusa. A Representaçâo de Afrodite na Lírica de Safo, Campinas: Editora Unicamp.

Reale, G., 1998. Platone. Fedro, Roma - Milano: Fondazione Lorenzo Valla. Mondadori.

Rissman, L., 1983. Love as war: Homeric allusion in the poetry of Sappho, Königstein/ Ts.: A. Hain. 
Robiano, P., 2003. "Maladie d'amour et diagnostic médical : Érasistrate, Galien et Héliodore d'Émèse ou du récit au roman". Ancient Narrative, 3, 129-49.

Rosenmeyer, P. A., 1997. "Her Master's Voice: Sappho's Dialogue with Homer". Materiali e Discussioni per l'analisi dei testi classici, 39, 129-49.

Russell, D. A., 1982. On the sublime, Oxford: at the Clarendon Press.

Schrenk, L. P., 1994. "Sappho Frag. 44 and the 'Iliad". Hermes, 122, 144-50.

Segal, C. P., 1996. "Eros and Incantation", in Re-Reading Sappho. Reception and

Transmission, ed. GreEne, E. Berkeley - Los Angeles - London, 58-75.

SteinRÜCK, M., 1999. "Homer bei Sappho". Mnemosyne, 52, 139-49.

ThÉvenaz, O., 2010. Sappho à Rome. Poétiques en échos de Catulle à Horace, Lausanne:

UNIL: thèse inédite.

Tzamal, E., 1996. Synatx und Stil bei Sappho, Dettelbach: Röll.

Voigt, E. M., 1971. Sappho, Alcaeus, Fragmenta, Amsterdam: Athenaeum : Polak et Van Gennep.

Weiske, B. (ed.) (1820). Dionysii Longini De Sublimitate: graece et latine, London.

Yatromanolakis, D., 2007. Sappho in the making : the early reception, Cambridge, Mass. ; London: Harvard University Press.

BOUVIER, David. O frêmito de Safo.

RESUMO: Se é verdade que a compreensão de uma obra antiga é sempre influenciada e condicionada, entre outros fatores, pela história das traduções, interpretações, retomadas e reapropriações que lhe garantiram a transmissão e recepção, essa constatação é particularmente verdadeira no caso da poesia de Safo. Palavra feminina num mundo culturalmente dominado pelo masculino, marcada com o selo da diferença, a poesia de Safo foi explicada tanto quanto foi reinventada, recuperada e subvertida. Mas, se sua poesia é indissociável da complexa história de sua recepção, a própria Safo se apropriou de uma tradição, senão de tradições, para cuja transformação ela colaborou.

PALAVRAS-CHAVE: Safo; frêmito; recepção; tradição. 